\title{
Local Group surveys for Planetary Nebulae
}

\author{
Laura Magrini ${ }^{1}$ \\ ${ }^{1}$ INAF-Osservatorio Astrofisico di Arcetri, Firenze, Largo E. Fermi, 5, 50125 Italy \\ email: laura@arcetri.astro.it
}

\begin{abstract}
The Local Group (LG) represents the best environment to study in detail the PN population in a large number of morphological types of galaxies. The closeness of the LG galaxies allows to investigate the faintest side of the PN luminosity function and to detect $\mathrm{PNe}$ also in the less luminous galaxies, the dwarf galaxies, where a small number of them is expected.

A review of the results of the most recent imaging surveys in the LG is presented. Some applications of the surveys for PNe to the study of the star formation history of the host galaxies are analyzed. In addition, these new observational data are an invaluable resource for followup spectroscopy to derive the chemical properties of not only $\mathrm{PNe}$, but also other important emission-line sources like HII regions. These are fundamental tools for the discussion of the chemical evolution of the host galaxies, mapping the history of their chemical enrichment at different epochs. The latest results on this subject are presented.
\end{abstract}

Keywords. planetary nebulae: general, Local Group, ISM: abundances

\section{The Local Group of galaxies}

The Local Group (LG) is composed of about 40 members. Their belonging to the LG is established using the following criteria (van den Bergh 2000): i) the distance to the galaxy should be $\leqslant 1.5 \mathrm{Mpc}$; ii) the galaxy should have a radial velocity which indicates that it might be be dynamically bound to the LG; iii) it should not appear to be associated with a group of galaxies that is know to be located well beyond the limits of the LG.

The two more massive galaxies, the spiral galaxies M31 and Milky Way, constitute the barycentres of two main subgroups. The Andromeda subgroup contains M31, its dwarf elliptical and spheroidal companions, among them the brightest ones are M32, NGC205, NGC185, NGC147, the late-type spiral galaxy M33, and many other dwarf galaxies, as IC10, DDO 210, SagDIG, Cas dSph. The Galaxy subgroup is composed by the Milky Way, the two irregular galaxies LMC and SMC, and a large number of dwarf spheroidal galaxies, like Draco, Ursa Minor, Fornax, Carina, LeoI, LeoII, Sculptor, Sagittarius. In addition, a number of isolated galaxies are present, like the dwarf irregular galaxies NGC 6822, IC 1613, WLM and Leo A. In the outskirts of the LG, a small association of dwarf galaxies (including NGC 3109, Antlia, Sextans A and B), represents the closest external group of galaxies. This group is not dominated by giant galaxies. Due to their proximity, the LG galaxies have been subject of deep and detailed studies (see the reviews by van den Bergh 2000, Mateo 1998). The structure of the LG, a small cluster containing essentially all morphological types of galaxies, except giant elliptical, is typical in the Local Universe. Consequently the results obtained from these galaxies might be extrapolated and generalized for farther galaxies. 


\section{The search for $\mathrm{PNe}$ in the $\mathrm{LG}$}

\subsection{A brief history of the search for PNe in the $L G$}

The first extragalactic (and LG) PN was discovered by Miss Annie Cannon. In her catalogue of peculiar spectra in the Magellanic Clouds (Cannon 1933), she also classified emission-line stars with [O III] 5007, 4959 and $\mathrm{H} \beta$. At first, these objects were not identified as PNe, while later some of them were recognized to be PNe.

The first efforts addressed to the detection of PNe in the LG were realized by Baade (1955) with the discovery of five PNe in M31 and contemporaneously by Lindsay (1955) with $17 \mathrm{PNe}$ in the SMC. Afterward, during the 60s and the early 70s the search was limited to the Magellanic Clouds, since the larger distances of the other galaxies limited the investigations. A review by Westerlund (1968) described the status at that time with $45 \mathrm{PNe}$ in LMC and 30 in SMC discovered by several investigators. Subsequently, in the 70 s and 80 s a series of papers, primarily by Ford, Jacoby and collaborators, reached other LG galaxies: NGC185, NGC205, M32 (Ford et al. 1973), NGC147 (Ford et al. 1977), Fornax (Danziger et al. 1978), IC10, LeoA, Sextans A, Pegasus, and WLM (Jacoby \& Lesser 1981) and discovered new PNe in M31 (Ford \& Jacoby 1978), LMC and SMC (Jacoby 1980). Only with the advent of CCD photometry, a large number of PNe was discovered in M31 and in its companions (Ciardullo et al. 1989). These authors used for the first time the PN luminosity function as a standard candle of extragalactic distances. This issue represented the main topic of the study of extragalactic PNe during the following decade, moving the attention from the LG to the giant galaxies beyond the LG where a larger PN population could be found. During the first surveys for PNe, M33 was partially neglected. An objective-prism survey for emission-line objects was attempted by Lequeux et al. (1987), but the first deep survey for PNe was obtained only in 2000 by Magrini et al. (2000). At the present time, M31 is the LG galaxy with the largest number of candidate PNe. In fact a new instrument, dedicated to the study of extragalactic PNe, the Planetary Nebula Spectrograph (Douglas et al. 2002), allowed to discover there $2615 \mathrm{PNe}$ and at the same time to measure their radial velocities (Merrett et al. 2006).

\subsection{The surveys of $L G$ galaxies $2001-2006$}

Currently, a new attention for the PN population in the LG has revived. During the last five years, two main surveys of the LG galaxies have been carried out: the Local Group Survey (P.I. P. Massey) and the Local Group Census (P.I. N. Walton).

The main aim of the 3-year survey Local Group Survey (LGS) had been to study the galaxies currently active in star-formation in the LG (Massey et al. 2002). The galaxies observed by the LGS are (in brackets the number of fields when greater than one): M31(10), M33(3), NGC 6822, IC 1613, IC 10, WLM, Pegasus, and Phoenix, and also Sextans A and Sextans B, located in the outskirts of the LG. These galaxies have been observed with he CTIO and KPNO 4-m telescopes equipped with the wide-field mosaic cameras (a mosaic of $8 \mathrm{k} \times 8 \mathrm{k}$ CCDs) using broad and narrow-band filters: UBVRI, $\mathrm{H} \alpha$, [S II] 671.7,673.1 nm, and [O III] $500.7 \mathrm{~nm}$. More information about this survey, the publications, the data release, can be found at:

$$
\text { http://www.lowell.edu/users/massey/lgsurvey.html }
$$

The Local Group Census (LGC) (2001-2003, 45 nights) has provided deep broad- and narrow-band images of LG galaxies with Dec $\geqslant-30^{\circ}$ (Corradi \& Magrini 2005), with the important exception of $\mathrm{M} 31$, for which observations aimed at detecting PNe had already been obtained by other ING programs. Observations were carried out using the Wide Field Camera (WFC) of the 2.5m INT telescope of the Isaac Newton Group of 
Telescopes at La Palma. The WFC is equipped with a mosaic of four $2 \mathrm{k} \times 4 \mathrm{k}$ CCDs covering a field of view of $34^{\prime} \times 34^{\prime}$. The pixel scale is $0 .^{\prime \prime} 33$. The galaxies observed by the LGC are (in brackets the number of WFC fields): Draco (4), DD210, EGB 0427+63, GR 8, IC 10, IC 1613, Leo A, Leo II, Leo I, M 33 (7), NGC 147, NGC 185, NGC 205 (2), NGC 6822 (4), Pegasus, Sextans A, Sextans B, Ursa Minor (4), and WLM. The majority of the galaxies were observed in four narrow-band filters [O III] $500.7 \mathrm{~nm}, \mathrm{H} \alpha+[\mathrm{N} \mathrm{II}$, [S II] $671.7,673.1 \mathrm{~nm}$, and He II $468.6 \mathrm{~nm}$. In addition, broad-band images were obtained using the Sloan $g^{\prime}, r^{\prime}, i^{\prime}$ and the Strömgren $Y$ filters. These filters have been used as a continuum for the narrow-band images to detect emission-line objects. In particular the Strömgren $Y$ and $g^{\prime}$ filters were used for the narrow-band filter [O III] $500.7 \mathrm{~nm}$ and $r^{\prime}$ for $\mathrm{H} \alpha+[\mathrm{N} \mathrm{II}]$. Typical exposure times were $60 \mathrm{~min}$ for the narrowband filters, $30 \mathrm{~min}$ for Strömgren $Y$, and 20 min for the Sloan filters. The completeness reached in the search for unresolved emission-line objects in relatively crowded regions of galaxies has been approximately $\mathrm{m}_{[O I I I]} \sim 24.5 \mathrm{mag}$, where $\mathrm{m}_{[O I I I]}$ is defined by Jacoby (1989). More details about the survey and a complete list of the data obtained can be found at:

http://www.ing.iac.es/ rcorradi/LGC/

Both surveys leaded up to a significant increase of the number of candidate PNe in LG galaxies. From 2000 to the present year the number of candidate PNe identified in the LG has increased of 100\% from 1500 to 3000 (excluding the Magellanic Clouds and the Galactic PNe, see Parker 2006). The data from the LGS observations were used by Ciardullo et al. (2004) to identify $152 \mathrm{PNe}$ in M 33. The results obtained until now by the LGC team in the search for PNe are: Sextans B (5 PNe) (Magrini et al. 2002), Sextans A (1), IC10 (16), LeoA (1) (Magrini et al. 2003B), NGC205 (35), NGC185 (5), NGC147 (9) (Corradi et al. 2005), NGC6822 (17) (Leisy et al. 2005), IC1613 (2), WLM (1), GR8 (0) (Magrini et al. 2005B). In particular, the PNe discovered by the LGC represent the first step in the study of the PN population in low-metallicity, dwarf irregular galaxies, since so far the PN population in these galaxies has been neglected.

\subsection{The identification techniques and criteria}

The most common techniques used to search for PNe in external galaxies are the onband off-band technique and the colour-colour diagrams. In addition, in wide intracluster regions automated detection algorithms have been directly applied to the continuumsubtracted frames (Feldmeier et al. 2003).

The techniques used by the LGC are described in the following. In order to search for emission-line objects in the LG galaxies, we subtracted from the [O III] frames the properly scaled Strömgren $Y$ or $g^{\prime}$ frames, choosing for each galaxy the best quality continuum. Similarly, the $r^{\prime}$ frames were subtracted from the $\mathrm{H} \alpha$ images. $\mathrm{H} \alpha-r^{\prime}$ vs. [O III $]-g^{\prime}$ diagrams were also obtained. The colour-colour diagrams are very powerful to detect unresolved sources in uncrowded sky regions, like the external regions of galaxies and the intracluster areas (Arnaboldi et al. 2003), while the continuum-subtracted images are proved to be more effective in crowded zones like the inner regions of galaxies Corradi et al. 2005).

The criteria used in the imaging searches to identify a "bona fide" candidate $\mathrm{PNe}$ are the following: i) they must appear in both the [O III] and $\mathrm{H} \alpha+[\mathrm{N} \mathrm{II}]$ images, but not in the continuum frames; ii) they must be unresolved at the distance of LG galaxies, taking into account the typical physical size of PNe (0.1-1 pc); iii) they should show the excitation parameter $\mathrm{R}=[\mathrm{O} \mathrm{III}] / \mathrm{H} \alpha+[\mathrm{N}$ II $]>1$ or somewhat larger (Magrini et al. 2000. Ciardullo et al. 2002) to distinghuish between compact HII regions and PNe in a statistical sense (but see, for example, the Type I PN in Sextans A, Magrini et al. 2005A); 


\begin{tabular}{|c|c|c|c|c|c|c|c|}
\hline Name & $\mathrm{T}$ & $\log \mathrm{L}_{V}$ & $\begin{array}{r}\mathrm{D} \\
{[\mathrm{kpc}]}\end{array}$ & $\begin{array}{c}\mathrm{N} . \\
\mathrm{PNe}\end{array}$ & Reference & $\begin{array}{l}\text { N. } \\
\text { S. }\end{array}$ & Reference \\
\hline M31 & $\mathrm{Sb}$ & 10.43 & 760 & 2615 & Merrett et al. 2006 & 30 & Jacoby \& Ciardullo 1999 \\
\hline M33 & $\mathrm{Sc}$ & 9.51 & 795 & 152 & Ciardullo et al. 2004 & 26 & Magrini et al. 2003A \\
\hline LMC & Ir & 9.35 & 50 & 1000 & Reid \& Parker 2005 & 141 & Leisy \& Dennefeld 2006A \\
\hline SMC & Ir & 8.79 & 59 & 132 & Jacoby 2005 & 42 & Leisy \& Dennefeld 2006A \\
\hline M32 & $\mathrm{E} 2$ & 8.55 & 760 & 46 & Merrett et al. 2006 & 14 & Richer \& McCall 2002 \\
\hline NGC205 & Sph & 8.51 & 760 & 35 & Corradi et al. 2005 & 13 & Richer \& McCall 2002 \\
\hline $\mathrm{IC} 10$ & $\mathrm{Ir}$ & 8.47 & 660 & 16 & Magrini et al. 2003B & - & Magrini et al. 2006 \\
\hline NGC6822 & $\mathrm{dIr}$ & 8.35 & 500 & 17 & Leisy et al. 2005 & 17 & $\begin{array}{l}\text { Leisy et al. 2006B } \\
\text { Hernandez \& Peña } 2006\end{array}$ \\
\hline NGC185 & Sph & 8.19 & 660 & 5 & Corradi et al. 2005 & 5 & Richer \& McCall 2002 \\
\hline $\mathrm{IC} 1613$ & $\mathrm{dIr}$ & 8.07 & 725 & 2 & Magrini et al. 2005B & - & Corradi et al. 2006 \\
\hline NGC147 & Sph & 7.99 & 660 & 9 & Corradi et al. 2005 & 8 & Gonçalves et al. 2006 \\
\hline WLM & $\mathrm{dIr}$ & 7.61 & 925 & 1 & Magrini et al. 2005B & - & \\
\hline Sagitt. & $\mathrm{dSp}$ & 7.47 & 24 & 4 & Zijlstra et al. 2006 & 4 & Zijlstra et al. 2006 \\
\hline Fornax & $\mathrm{dSp}$ & 7.19 & 138 & 1 & Danziger et al. 1978 & 1 & Danziger et al. 1978 \\
\hline Pegasus & dIr & 6.87 & 760 & 1 & Jacoby \& Lesser 1981 & - & \\
\hline LeoA & $\mathrm{dIr}$ & 6.55 & 690 & 1 & Magrini et al. 2003B & 1 & van Zee et al. 2006 \\
\hline NGC3109 & dIr & 8.27 & 1330 & 13 & $\begin{array}{l}\text { Leisy et al. 2006B } \\
\text { Peña et al. 2006 }\end{array}$ & 12 & $\begin{array}{l}\text { Leisy et al. 2006B } \\
\text { Peña et al. } 2006\end{array}$ \\
\hline SextansB & $\mathrm{d} I \mathrm{r}$ & 7.63 & 1600 & 5 & Magrini et al. 2002 & 5 & Magrini et al. $2005 \mathrm{~A}$ \\
\hline SextansA & $\mathrm{dIr}$ & 7.67 & 1320 & 1 & Magrini et al. 2003B & 1 & Magrini et al. $2005 \mathrm{~A}$ \\
\hline
\end{tabular}

Table 1. The Local Group and outskirts galaxies with PNe detection. The morphological types (T), luminosities ( $\log L_{V}$ and distances (D) are from van den Bergh (2000). The references to the number of candidate PNe (N.PNe) and to their spectroscopic confirmation (N. S.) are in the adjacent columns.

iv) the candidate PNe in the top 1 mag of the PNLF should have generally $\mathrm{R} \geqslant 2$ (see Fig. 2 of Ciardullo et al. 2002).

Table 1 shows the galaxies with detected candidate PNe belonging to the LG and to its outer fringes. The morphological types, the luminosities, the distances are from van den Bergh (2000). The references to the latest identification of $\mathrm{PNe}(\mathrm{N}$. PNe) and to their spectroscopic confirmation (N. S.) are in the adjacent columns. The search for PNe is almost complete within the first 2-3 magnitude of their luminosity function for all the galaxies where a PNe population is expected $\left(\log \mathrm{L}_{v} \geqslant 6.7\right.$, Magrini et al. 2003B). On the other hand, the spectroscopic confirmation has been obtained for only $10 \%$ of them (see Table1).

\section{What can we learn from Local Group PNe (and HII regions)?}

Why LG PNe are so special and why do we want to study them in detail? They have the advantage to be located outside the Galaxy, and consequently to have well known distances. On the other hand they are relatively close. Thus, among the extragalactic $\mathrm{PNe}$, physical and chemical properties can be derived measuring directly electron temperature and densities only for them. Furthermore, they belong to a variegated sample of galaxies with different morphology, metallicity, chemical evolution, and star formation history. In the following sections, some examples of their application to the study of LG galaxies are described. 


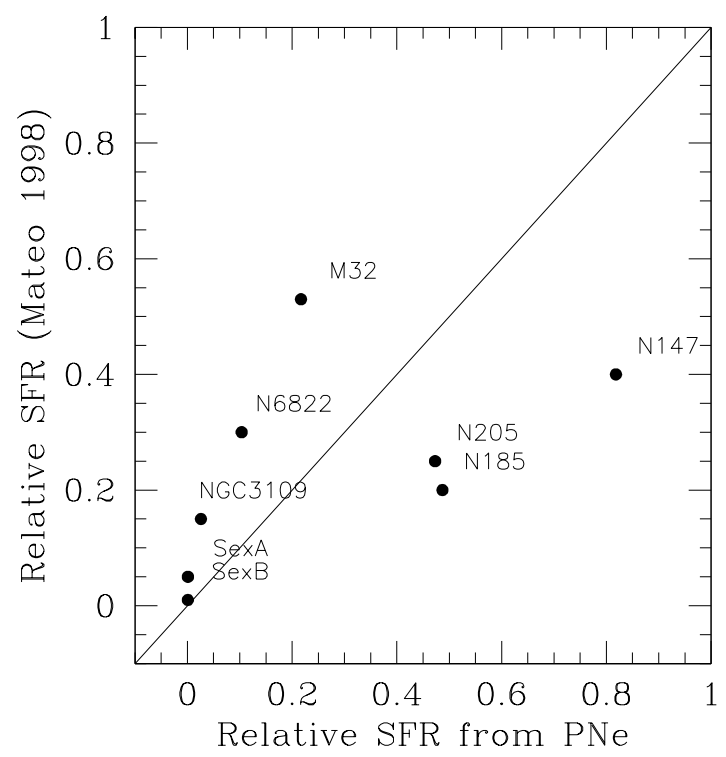

Figure 1. The relative SFR in the intermediate-age for LG galaxies: computed from the number of PNe extrapolated to 4 mag below the bright end of the PNLF (x axes) and the SFR from Mateo (1998) (y axes).

\subsection{Photometry}

The PN population size, when corrected for the different completeness limits of the various surveys, scales well with the V luminosity of a galaxy (Magrini et al. 2003B Corradi \& Magrini 2005), but it is expected to be sensitive to the relative amount of star formation during the epoch of the formation of the PN progenitors, the last 1 to 8 Gyr (the so called intermediateage). In fact, their number reflects the total mass of the underlying stellar population from which they derive. The total mass of the progenitor population can be derived using the theoretical relation between the total luminosity of the population of $\mathrm{PNe}$ progenitors and the PNe number, obtained under the hypothesis of coeval, chemically homogeneous stars (Renzini \& Buzzoni 1986). The total luminosity of the population of PNe progenitors, $L_{T_{\mathrm{PPNe}}}$, is related to the number of PNe, $n_{\mathrm{PNe}}$, to their lifetime, $t_{\mathrm{PNe}}$, and to the so-called evolutionary flux, $\dot{\xi}_{\mathrm{PNe}}$. The number of stars with initial mass $0.8 M_{\odot}<M<8 M_{\odot}$ per unit luminosity leaving the Main Sequence each year is then given by the expression

$$
L_{T_{\mathrm{PPNe}}}=\frac{n_{\mathrm{PNe}}}{t_{\mathrm{PNe}} \dot{\dot{\xi}_{\mathrm{PNe}}}} .
$$

Thus, counting planetary nebulae, extrapolating their number to the same completeness limit, i.e. 4 mag below the bright end of the PNLF, and estimating the mean mass of the PNe progenitors, we have a measure of the total mass of the intermediate-age stellar population (Magrini et al. 2005B). The intermediate-age stellar mass is thus proportional to the number of PNe: for each PN observed a total mass of $\sim 2 \times 10^{6} \mathrm{M}_{\odot}$ composed by stars with initial mass $0.8 M_{\odot}<M<8 M_{\odot}$ is formed. Considering the whole IMF (i.e. Scalo 1998) we have that $10^{7} \mathrm{M}_{\odot}$ were produced for each PNe observed. In Figure 1, we show the SFR known from the literature (cf. (Mateo 1998), vs. that computed with PNe, from the ratio of the mass of intermediate-age population and the total stellar mass. The solid line is the bisector of the first quadrant. A good agreement between the two SFRs 
can be noticed. The most remarkable exception is NGC147, where the SFR derived from $\mathrm{PNe}$ is higher than expected. PNe are therefore confirmed to be useful evolutionary agetracers of the intermediate-age population. The presence of PNe is a good and valuable evidence for an intermediate-age population (cf. Aparicio \& Gallart 1994). Moreover the relationship of their number to the host galaxy mass gives quantitative information on the relative star formation rate.

\subsection{Spectroscopy}

The elemental abundances measured in PNe are extremely important to investigate the metal content of the host galaxy a few Gyrs ago, at the time when their stellar progenitors formed. In fact, the PN progenitor do not process elements like $\mathrm{S}$ and Ar, and to a first approximation also $\mathrm{Ne}$ and $\mathrm{O}$; therefore these abundances in $\mathrm{PNe}$ probe the metallicity of the interstellar medium when their progenitor stars were born. On the other hand, the progenitors of $\mathrm{PNe}$ produce and return to the interstellar medium elements like He, C, and N. Therefore, measuring abundances of these elements allows to test stellar evolution models. The PNe in the LG have known distances, and thus the luminosity and temperature of their central stars can be estimated via modelling with CLOUDY, as done in Magrini et al. (2005A). The masses of the PN central stars, and hence the mass and age of their progenitors, can then be calculated from the central stars location on the Hertzprung-Russell diagram. Completing the study of the samples of PNe with HII regions allows us to plot the age-metallicity relationship and thus to study the chemical enrichment of the host galaxy (see Figure 2).

As an example of this kind of study, we report the results obtained with the VLT spectroscopy of the PN and HII region populations in Sextans A and Sextans B (Magrini et al. 2005A). The main results are:

i) The $\mathrm{O} / \mathrm{H}$ abundance of HII regions is homogeneous throughout the galaxies, contrary to the large dishomogenities expected due to the episodic star formation and to the solid body rotation of dIr galaxies (Skillman et al. 1989).

ii) The age-metallicity relation is almost constant for both galaxies, indicating a very low (or absent) chemical enrichment in these galaxy in the last 10 Gyr (see Figure 2).

iii) The present time $\mathrm{O} / \mathrm{H}$ abundances from HII regions of Sextans A and Sextans B are comparable within the errors, $12+\log (\mathrm{O} / \mathrm{H})=7.6 \pm 0.2$ for Sextans $\mathrm{A}$ and $7.8 \pm 0.2$ for Sextans B (Magrini et al. 2005A), in spite of quite different star formation histories. This result confirms that galaxies with similar luminosity and morphological type have also similar metallicity, and maintain it over a large time interval. This means that the wellknown metallicity-luminosity relationship represents the ability of a galaxy to keep the product of its own evolution more than to produce metals.

$i v)$ In low metallicity stars the nucleosynthesis and dredge-up processes are predicted to be more efficient than in solar metallicity stars (i.e. Herwig 2004 Marigo 2001), but observations of Galactic PNe to test the theoretical models are still missing. The oxygen overabundance of the only PN known in Sextans A compared with that of HII regions in the same galaxy, suggests oxygen enrichment due to the third-dredge-up process for massive $\left(\mathrm{M}>3 \mathrm{M}_{\odot}\right)$, low-metallicity PNe progenitors. This is in good agreement with the predictions of the above quoted theories (Marigo et al. 2004).

These results show how $\mathrm{PNe}$ and HII regions are of great importance to set constraints to chemical evolution models of dwarf galaxies (see above i, ii, iii) (cf. Hensler et. 1999

Recchi et al. 2001) and also to study the nucleosynthesis of low-metallicity stars (iv).

\section{Acknowledgments:}

I would thank the SOC of this Conference for having invited me and for the IAU grant. It is a pleasure to thanks the LGC team for the work done together during these years. 


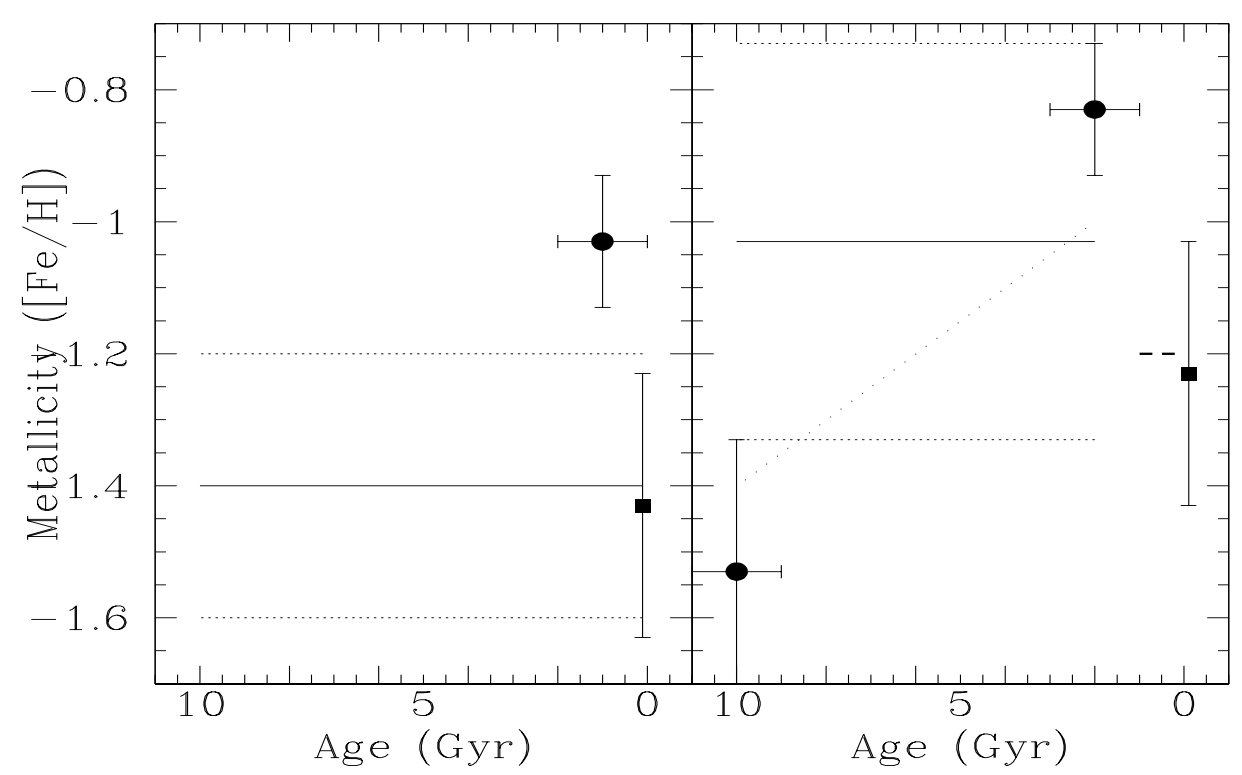

Figure 2. The age-metallicity relationship for the dIr galaxies Sextans A (left panel) and Sextans B (right panel). For Sextans A: the solid line is the mean $[\mathrm{Fe} / \mathrm{H}]$ of stars derived from colour-magnitude diagrams (CMD) by Dolphin et al. (2003), while the dotted lines represent the spread of stellar metallicities. The square is the mean of metallicity of the HII regions and the error bars are the rms scatter, the filled circle is the PN abundance. For Sextans B: the solid is the mean abundance of the Sextans B PNe with its rms scatter (dotted lines), the filled circles are the abundances of the oldest and the youngest PNe. The square is the mean metallicity of the HII regions and the error bars are the rms scatter. The dashed line is the stellar metallicity derived by CMD by Tosi et al. (1991). The transformation from [O/H] to $[\mathrm{Fe} / \mathrm{H}]$ needed to compare our nebular oxygen abundances with the stellar abundances was computed according to the empirical transformation by Mateo $(1998):[\mathrm{Fe} / \mathrm{H}]=[\mathrm{O} / \mathrm{H}]-0.37$, where $[\mathrm{O} / \mathrm{H}]=\log (\mathrm{O} / \mathrm{H})-\log (\mathrm{O} / \mathrm{H})_{\odot}$. We used the solar abundances from Asplund (2003) to derive $[\mathrm{O} / \mathrm{H}]$ from the measured $12+\log (\mathrm{O} / \mathrm{H})$.

\section{References}

Aparicio A., Gallart C., 1994, in The Local Group: Comparative and Global Properties, ESO Conference and Workshop Proceedings, Eds Andrew Layden, R. Chris Smith, and Jesper Storm, p.115

Arnaboldi, M., Freeman, K. C., Okamura, S., et al. 2003, AJ, 125, 514

Asplund, M. 2003, CNO in the Universe, ed. C. Charbonnel, D. Schaerer, G. Meynet (San Francisco: ASP), ASP Conf. Ser., 304, 275

Baade, W., 1955, AJ, 60, 151

Cannon, A. J., 1933, HCOB, 891, 2

Ciardullo, R., Jacoby, G.H., Ford, H.C., Neill, J.D. 1989, ApJ, 339, 53

Ciardullo R., Feldmeier J.J., Jacoby G.H., Kuzio de Naray R., Laychak M., Durrell P.R., 2002, ApJ, 577, 31

Ciardullo, R., Durrell, P. R., Laychak, M. B., et al. 2004, ApJ, 614, 167

Corradi, R.L.M., Magrini, L., 2005, in the proceedings of the ESO-Workshop "Planetary Nebulae beyond the Milky Way", Eds. L. Stanghellini, J.R. Walsh, N.G. Douglas, p.36

Corradi, R.L.M., Magrini, L., Greimel, R., et al. 2005, A\&A, 431, 555

Corradi, R.L.M., Magrini, L., Leisy, P., et al. 2006, in preparation

Danziger, I.J., Webster, B.L., Dopita, M.A., Hawarden, T.G, 1978, ApJ, 220, 458

Dolphin, A. E., Saha, A., Skillman, E. D., et al., 2003, AJ, 126, 187

Douglas, N. G.; Arnaboldi, M.; Freeman, K. C., et al., PASP, 114, 801, 1234 
Feldmeier, J. J., Ciardullo, R., Jacoby, G. H., \& Durrell, P. R., 2003, ApJS, 145, 65

Ford, H.C., Jenner, D.C., Epps, H.W., 1973, AJ, 183, 73

Ford, H.C., Jacoby, G.H., Jenner, D.C., 1977, ApJ, 213, 18

Ford, H.C., Jacoby, G.H. 1978, ApJS, 38, 351

Gonçalves, R.D., Magrini, L., Leisy, P., Corradi, R., 2006, this book

Hensler, G., Rieschick, A., Koepen, J.,1999, ASPC, 187, 214

Hernandez-Martinez, L., Peña, M., 2006, this book

Herwig, F., 2004, ApJS, 155, 651

Jacoby, G.H., 1980, ApJS, 42, 1

Jacoby, G.H., 1989, ApJ, 339, 39

Jacoby, G.H., 2005, in the proceedings of the ESO-Workshop "Planetary Nebulae beyond the Milky Way", Eds. L. Stanghellini, J.R. Walsh, N.G. Douglas, p.17

Jacoby, G. H., Ciardullo, R., 1999, ApJ, 515, 169

Jacoby, G.H., Lesser, M.P., 1981, AJ, 86, 185

Leisy, P., Dennefeld, M., 2006, A\&A in press

Leisy, P., Magrini, L., Corradi, R., 2006, in preparation

Leisy, P., Corradi, R.L.M., Magrini, L., Greimel, R., Mampaso, A., Dennefeld, M., 2004, A\&A, 436,437

Lequeux, J., Meyssonnier, N., Azzopardi, M., 1987, A\&AS, 67, 169

Lindsay, E.M., 1955, MNRAS, 115, 248

Magrini L., Corradi, R.L.M., Mampaso, A., Perinotto, M. 2000, A\&A, 355, 713

Magrini, L., Corradi, R.L.M., Walton, N.A., Zijlstra, A. A., Pollacco, D.L., Walsh, J.R., Perinotto, M., Lennon, D.J., Greimel, R., 2002, A\&A, 386, 869

Magrini, L., Perinotto, M., Corradi, R. L. M., Mampaso, A., 2003, A\&A, 400, 511

Magrini, L., Corradi, R.L.M., Greimel, R., Leisy, P., Lennon, D.J., Mampaso, A., Perinotto, M., Pollacco, D.L., Walsh, J.R., Walton, N.A., Zijlstra, A.A., 2003, A\&A, 407, 51

Magrini, L., Leisy, P., Corradi, R.L.M., Perinotto, M., Mampaso, A., Vilchez, J.M., 2005A, A\&A, 443, 115

Magrini, L., Corradi, R.L.M., Greimel, R., et al., 2005B, MNRAS, 361, 517

Magrini, L., Corradi, R.L.M., Leisy, P., 2006, in prep.

Marigo, P., 2001, A\&A, 370, 194

Marigo, P., Girardi, L., Weiss, A., Groenewegen, M.A.T., Chiosi, C., 2004, A\&A, submitted

Mateo, M., 1998 A\&A Rev 36, 435

Massey, P., Hodge, P.W., Holmes, S., et al., 2002, AAS, 201, Vol. 34, p.1272

Merrett, H. R., Merrifield, M. R., Douglas, N.G., et al., 2006, astro-ph/0603125

Parker, Q.A., this book

Peña, M., Richer, M., Stasinska, G., 2006, this book

Recchi, S., Matteucci, F., D'Ercole, A,, 2001, MNRAS, 322, 800

Reid, W. \& Parker, Q.A., in the proceedings of the ESO-Workshop "Planetary Nebulae beyond the Milky Way", Eds. L. Stanghellini, J.R. Walsh, N.G. Douglas, p.30

Renzini, A., Buzzoni, A., 1986 in Spectral Evolution of Galaxies, eds. Chiosi C. and Renzini A., Reidel, Ap. Space Sci. Lib., Vol. 122, p.195

Richer, M. G., McCall, M. L., 2002, RMxAC, 12, 173

Scalo J.M., 1998, in The Stellar Initial Mass Function, ed, G. Gilmore, \& D. Howell (San Francisco:ASP), ASP Conf. Ser., 142, 201

Skillman, E. D., Kennicutt, R. C., Hodge, P. W., 1989, ApJ, 347, 875

Tosi, M., Greggio, L., Marconi, G., \& Forcardi, P. 1991, AJ, 102, 951

van den Bergh, S., 2000, in The Galaxies of the Local Group, (Cambridge: Cambridge University Press)

van Zee, L., Skillman, E. D., Haynes, M. P., 2006, ApJ, 637, 269

Westerlund, B.E., IAU Symp. n. 34, Osterbrock D.E., O’Dell C.R. eds., Dordrecht, p.23

Zijlstra, A., Gesicki, K., Walsh, J. R., Pequignot, D., van Hoof, P. A. M., Minniti, D., MNRAS accepted, astro-ph/0603422 